\title{
In Silico Docking Analysis of A-type Proanthocyanidins to a-Glucosidase Constructed by Correlation with in Vitro Bioassay
}

\author{
Sheau Ling Ho ${ }^{1}$, Yili Lin ${ }^{2}$, Shengfa Tsai ${ }^{2}$, Shoeisheng Lee ${ }^{2, *}$ \\ ${ }^{1}$ Department of Chemical \& Materials Engineering, Chinese Culture University, Taipei, Taiwan, ROC \\ ${ }^{2}$ School of Pharmacy, College of Medicine, National Taiwan University, Taipei, Taiwan, ROC \\ Email address: \\ hx13@faculty.pccu.edu.tw (Sheau Ling Ho),r03423003@ntu.edu.tw (Yili Lin), apha8@yahoo.com.tw (Shengfa Tsai), \\ shoeilee@ntu.edu.tw (Shoeisheng Lee) \\ ${ }^{*}$ Corresponding author
}

\section{To cite this article:}

Sheau Ling Ho, Yili Lin, Shengfa Tsai, Shoeisheng Lee. In Silico Docking Analysis of A-type Proanthocyanidins to $\alpha$-Glucosidase Constructed by Correlation with in Vitro Bioassay. Journal of Drug Design and Medicinal Chemistry. Vol. 5, No. 4, 2019, pp. 47-60.

doi: $10.11648 /$ j.jddmc.20190504.11

Received: November 21, 2019; Accepted: December 7, 2019; Published: December 23, 2019

\begin{abstract}
The type A proanthocyanidins $(2-8)$ with $(2 \beta \rightarrow \mathrm{O} \rightarrow 7,4 \beta \rightarrow 8)$ interflavane linkage, isolated from Machilus philippinensis, have been found to possess inhibitory activity against $\alpha$-glucosidase (EC 3.2.1.20 from Bacillus stearothermophilus). To rationalize such activity, computer assisted docking of these compounds and the positive control, acarbose, on the conformation model of $\alpha$-glucosidase (AG), built by using human intestinal maltase glucoamylase as a template, was undertaken in this study. The result showed good correlation between $\mathrm{IC}_{50}$ values and docking scores, expressed as binding energy $(\Delta G)$, obtained from London (trimatch)-refinement (forcefield Affinity $\Delta G$ ) mode. Among these isolates, the most potent aesculitannin B (2) $\left(\mathrm{IC}_{50} 3.5 \mu \mathrm{M}\right)$ showed the best docking score $(\Delta G-21.48 \mathrm{kcal} / \mathrm{mol})$. Being interested in clarification of structure and activity relationship, virtual screening on the related compounds, including the de-unit III homologs of 2-8 (i.e., nor- series) and additional 13 stereoisomers of the trimeric 2 at the C-2 and C-3 positions of units II and III, was further carried out. This docking study indicated the de-unit III homologs of 2-8 did not have better binding energies than 2 . As for the trimers, 3-ent C, 3C-entE, 3ent-C, 3C, and 3ent, showed comparable docking score to 2 . The verification of this virtual screening was partially done by evaluating the inhibitory activity of the dimeric 2-nor-ent, 3-nor, 3-nor-ent, and iso-2-nor-ent, isolated from peanut skins, against $\alpha$-glucosidase. Of these, iso-2-nor-ent, the only proanthocyanidin with $(2 \beta \rightarrow \mathrm{O} \rightarrow 7,4 \beta \rightarrow 6)$ interflavane linkage, showed the best activity $\left(\mathrm{IC}_{50} 9.72 \mu \mathrm{M}\right)$. Their simulation profiles of docking score also displayed a reasonable qualitative consistency with the overall trend of the bioassay results. This study demonstrates that virtual screening using this built model to search $\alpha$-glucosidase inhibitors is facile and feasible and peanut skin might be used as a hypoglycemic food.
\end{abstract}

Keywords: $\alpha$-Glucosidase, Proanthocyanidins, Bioassay, Molecular Docking, Proanthocyanidin Homologs, Peanut Skin

\section{Introduction}

$\alpha$-Glucosidase (EC 3.2.1.20), an exo-type glycosidase, can digest oligosaccharide and disaccharide by hydrolyzing the $\alpha-1$, 4-glycosidic linkage to release glucose [1]. Maltase-glucoamylase (EC 3.2.1.20 \& 3.2.1.3), present in human small intestinal mucosa and exerting the $\alpha$-glucosidase activity, is the key enzyme involved in carbohydrate digestion and glucose absorption [2,3]. While this enzyme is inhibited, glucose absorption will be delayed, leading to the decrease of postprandial blood glucose level [4]. Such inhibitors including acarbose $\left(\right.$ Glucobay $\left.^{\circledR}\right)$ and voglibose $\left(\right.$ Voglib $\left.^{\circledR}\right)$ have been used in clinic for the treatment of hyperglycemia in type-2 diabetes mellitus patients. Recently, some potent $\alpha$-glucosidase inhibitors, such as oxindoles [5] and biscoumarins [6], have been developed. Our study found that isovitexin 2"-(E)-p-coumarate among 17 flavonoids isolated from Tinospora crispa leaf showed the best activity against $\alpha$-glucosidase with an $\mathrm{IC}_{50}$ value of $4.3 \pm 1.4 \mu \mathrm{M}$ [7]. The 3'-hydroxylated derivative, however, was much less active 
$\left(\mathrm{IC}_{50} 35.7 \mu \mathrm{M}\right)$. Such significant difference was rationalized by molecular dynamic (MD) simulation study on constructed $\alpha$-glucosidase (AG) model [7]. The good correlation between in vitro assay and MD simulation encouraged us to investigate whether the anti- $\alpha$-glucosidase proanthocyanidins (Figure 1), isolated from Machilus philippinensis leaf [8], follow such relationship. To elucidate the effect of unit III and the stereochemistry of $\mathrm{C}-2$ and $\mathrm{C}-3$ at units II and III on $\alpha$-glucosidase activity, the docking study on de-unit III homologs of 2-8 (Figure 1) and 13 trimeric proanthocyanidins, being stereoisomers of aesculitannin B (2) at the C-2 and C-3 positions in units II and III (Figure 2), was undertaken. For partial verification of the latter virtual docking, three A-type interflavanoid proanthocyanidins (2-nor-ent, iso-2-nor-ent, 3-nor, and 3-nor-ent) were re-isolated from peanut skin [9] and their anti- $\alpha$-glucosidase activity was assayed.

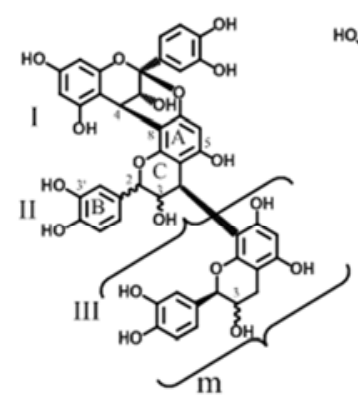

2: $m=1 ; I I-2 \alpha, 3 \beta ; I I I-3 \beta$ 3: $m=1 ;\|-2 \beta, 3 \beta ;\|-3 \beta$ 4: $m=1 ; I I-2 \beta, 3 \beta ; I I I-3 \alpha$ 2-nor: $m=0 ; \|-2 \alpha, 3 \beta$ 2-nor-ent: $\mathrm{m}=0 ; \|-2 \beta, 3 \alpha$ 3-nor: $m=0 ; 11-2 \beta, 3 \beta$ 3-nor-ent: $\mathrm{m}=0 ; 11-2 \alpha, 3 \alpha$
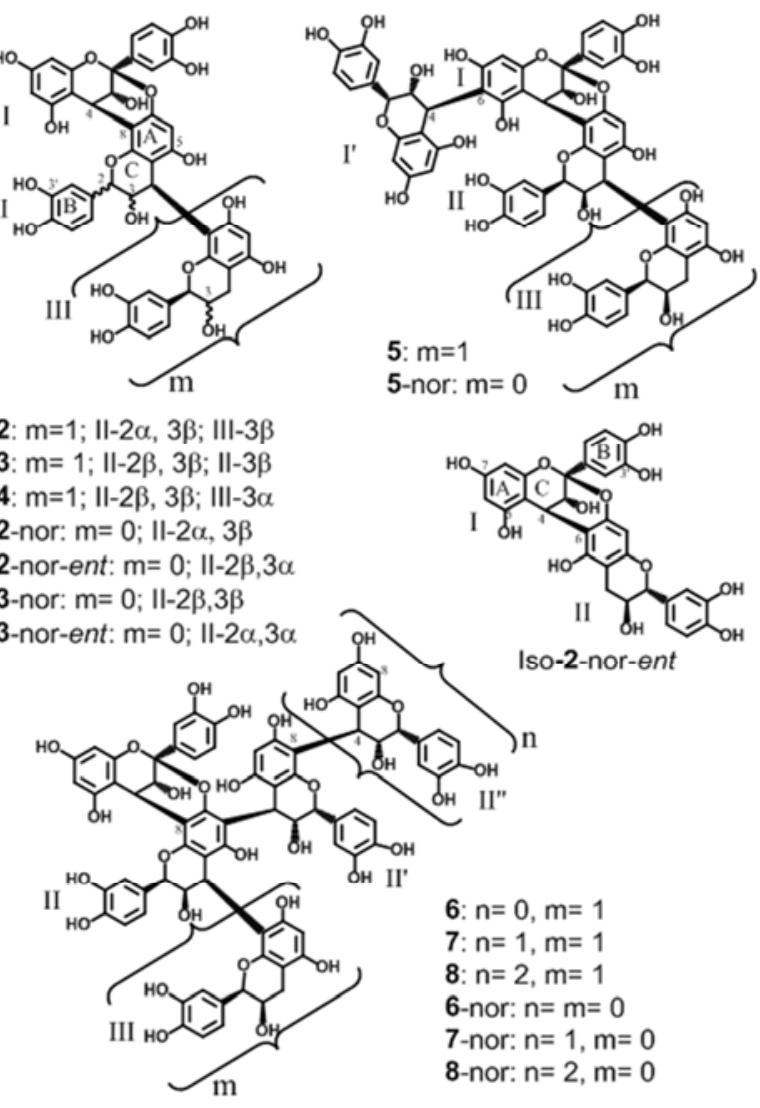

Figure 1. The structures of proanthocyanidins (2-8) isolated from Machilus philippinensis and their corresponding de-unit III analogs, and, an epimer of 2-nor (2-nor-ent)

\section{Materials and Methods}

\subsection{Molecular Dynamic (MD) Simulation}

\subsubsection{Construction of $\alpha$-Glucosidase Conformation as Docking Model}

The conformation model of $\alpha$-glucosidase (AG; EC 3.2.1.20 from Bacillus stearothermophilus) using human intestinal maltase glucoamylase (PDB ID: 2QLY) [10] as a template had been constructed as described in our recent publication [7]. All the computational and structural studies were carried out using MIFit (a cross-platform interactive graphics application for molecular modelling) and Molecular Operating Environment (MOE, 2010.10; Chemical Computing Group Inc. software) [11]. To model the binding sites of $\alpha$-glucosidase, the SiteFinder function of MOE based on the constructed three-dimensional $\alpha$-glucosidase was used.

The key catalytic sites, D518 and D616, and sequence motifs of family 31 glycosyl hydrolases were well conserved $[12,13]$. Like many other sugar-binding enzymes, there were a lot of hydrophobic residues lining the active-site pocket, including W376, W402, I441, W481, W516, F525, W613, and F649 [14].

\subsubsection{Ligands for Docking}

Seven A-type proanthocyanidins $(2-8)$ together with epicatechin (1), isolated from the EtOH extract of the leaves of Machilus philippinensis [8] and four dimeric isomers (2-nor-ent, iso-2-nor-ent, 3-nor, and 3-nor-ent), isolated from peanut skins [9], were chosen for this in silico docking analysis (Figure 1). Next, the docking of the truncated 2-8 (2-nor-8-nor), obtained by deleting unit III, and all possible trimeric stereoisomers at the $\mathrm{C}-2$ and $\mathrm{C}-3$ positions of units II and III of 2-4 (Figure 2) to AG were carried out. The three dimension structures of these compounds and acarbose were constructed using ChemBio3D, then imported them to MOE for molecular docking simulation.

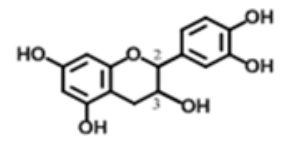

epicatechin $(1, E): 2 \alpha, 3 \alpha$ ent-epicatechin (entE): $2 \beta, 3 \beta$ catechin $(C): 2 \alpha, 3 \beta$

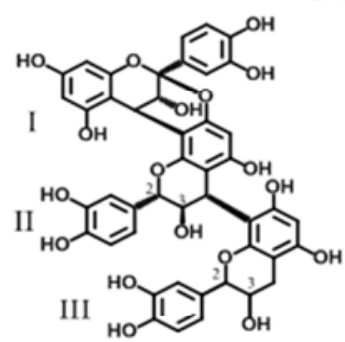
ent-catechin $(e n t C): 2 \beta, 3 \alpha$

$$
\begin{aligned}
& \text { 2: } 2 \beta, 3 \beta \\
& \text { 2-C: } 2 \beta, 3 \alpha \\
& \text { 2-entC: } 2 \alpha, 3 \beta \\
& \text { 2-entE: } 2 \alpha, 3 \alpha
\end{aligned}
$$

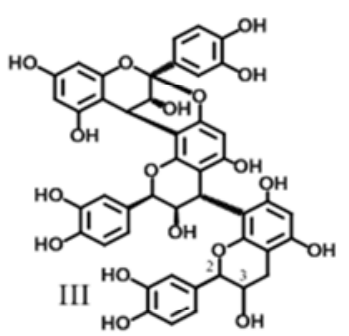

3: $2 \beta, 3 \beta$

$4(3-C): 2 \beta, 3 \alpha$

3-ent C: $2 \alpha, 3 \beta$

3-entE: $2 \alpha, 3 \alpha$

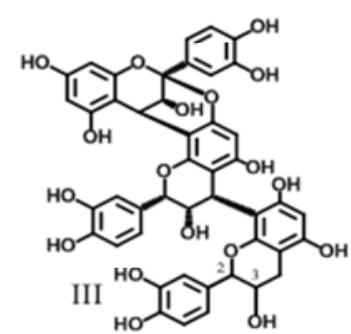

3ent-C: $2 \beta, 3 \alpha$

3ent: $2 \beta, 3 \beta$

3ent-entC: $2 \alpha, 3 \beta$

3ent-entE: $2 \alpha, 3 \alpha$

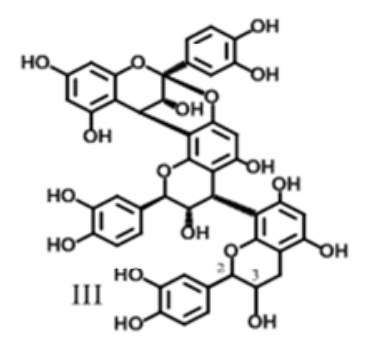

$3 C: 2 \beta, 3 \beta$

$3 C-C: 2 \beta, 3 \alpha$

$3 C$-entC: $2 \alpha, 3 \beta$

$3 C$-entE: $2 \alpha, 3 \alpha$

Figure 2. Stereoisomers of the trimeric proanthocyanidin 2 at the $C-2$ and $C-3$ chiral centers of units II and III for MD study. 


\subsubsection{Molecular Docking and Post-dynamic Analysis}

Molecular docking study was done to reveal the ligand-receptor interactions and to compare affinities of various compounds to the target AG model. The AG structure was protonated in the MOE [15]. The triangle matcher algorithm of the MOE software packages was selected to dock the identified hit compounds into the chosen protein active site. Docking calculations were carried out using standard default variables for the MOE. Binding affinity was evaluated by the binding energies (S-score, $\mathrm{kcal} / \mathrm{mol}$ ), hydrogen bonds, and root-mean-square deviation (RMSD) values. The binding energy was calculated between the protein and the ligand, intramolecular hydrogen bonds and strains of the ligand. The RMSD was computed in terms of all the atoms in a protein backbone and the value was less than $0.6 \AA$ which was indicative of considerable structural similarity. The compounds were docked into same groove of the binding site. Then, the initial model was loaded into MOE working environment ignoring water molecules and heteroatoms. The structure with all the atoms shown was put in generalized born implicit solvated environment at a temperature of $300 \mathrm{~K}, \mathrm{pH}$ of 7.0 , and a salt concentration of 0.1 $\mathrm{mol} / \mathrm{L}$. Electrostatic potential was applied to a cut-off value of $1.5 \AA$ at a dielectric value of 1.0. A non-bonded cut-off value of $5.0 \AA$ was applied to the Leonard-Jones terms. The dock scoring in MOE software was done using London $\Delta G$ scoring function to estimate the ligand-protein binding free energy and enhanced by the Forcefield refinement method (Affinity $\Delta G$ or London $\Delta G$ ) to relax the poses and then the refinement scores to rank the poses output to AG. Poses had been updated to ensure that refined poses satisfy the specified conformations. The rotatable bonds were allowed and then the best 20 poses were retained to analyze their binding scores. Energy minimization was conducted through Force-field MMFF94x optimization using a gradient cut-off value of 0.05 $\mathrm{Kcal} / \mathrm{mol} / \AA$ for determining low energy conformations with the lowest energy geometry [16]. From the final list of these 20 docked conformations, the pose with least docking score ligand was then chosen for further analysis.

\subsection{Isolation of Proanthocyanidins from Peanut Skin}

The dry peanut skins $(7.00 \mathrm{~kg})$ obtained from the baked peanut were stirred with $95 \% \mathrm{EtOH}(1 \times 45 \mathrm{~L}, 3 \times 24 \mathrm{~L})$ at $50^{\circ} \mathrm{C}$. The EtOH solutions were concentrated under reduced pressure at $50^{\circ} \mathrm{C}$ to give the $\mathrm{EtOH}$ extract $(1.62 \mathrm{~kg})$. The suspension of the EtOH extract $(250.20 \mathrm{~g})$ in $\mathrm{H}_{2} \mathrm{O}(2.5 \mathrm{~L})$ was partitioned in sequence against $\mathrm{CH}_{2} \mathrm{Cl}_{2}$, EtOAc, and $n$-BuOH (saturated with $\mathrm{H}_{2} \mathrm{O}$ ), each $3 \times 2.5 \mathrm{~L}$, to give fractions soluble in $\mathrm{CH}_{2} \mathrm{Cl}_{2}$ (78.4 g), EtOAc (42.6 g), $n$-BuOH (78.8 g), and $\mathrm{H}_{2} \mathrm{O}(30.3 \mathrm{~g})$ after evaporation of each fraction under reduced pressure at $50^{\circ} \mathrm{C}$.

An aliquot of the EtOAc-soluble fraction (28.6 g) was fractionated on a Sephadex LH-20 column (Pharmacia Co; 8 $\mathrm{cm}(\mathrm{OD}) \times 89 \mathrm{~cm}, \mathrm{MeOH})$ to give 11 fractions (E1 11), combined based on TLC analysis. Fraction E4 was further fractionated by centrifugal partition chromatograph (CPC; Model L.L.B-M, 230 mL; Sanki Engineering Ltd., Japan) using the $n-\mathrm{BuOH}-\mathrm{EtOH}-\mathrm{H}_{2} \mathrm{O}(10: 2.5: 10)$ system, flow rate $1.0 \mathrm{~mL} / \mathrm{min}$. Two aliquots of this fraction (E4, $999 \mathrm{mg}$ and 947 $\mathrm{mg}$ ) were delivered by the lower layer (water layer) with the rotation speed $1400 \mathrm{rpm}$ to give seven fractions (E4-A1 7). Another aliquot (E4, $1076 \mathrm{mg}$ ) was delivered by the upper layer ( $n$-BuOH layer) with the rotation speed $1600 \mathrm{rpm}$ to give seven fractions (E4-B1 7). Fractions E4-A3 (333 mg) and E4-B3 $(373 \mathrm{mg}$ ) were combined and was further fractionated by $\mathrm{CPC}$ delivered by the upper layer ( $n$ - $\mathrm{BuOH}$ layer), flow rate $0.5 \mathrm{~mL} / \mathrm{min}$ with the rotation speed $1800 \mathrm{rpm}$, to give five fractions $(\mathrm{E} 4-\mathrm{C} 1 \sim 5)$. Fractions E4-A4, B2, and C2 were combined to give a residue $(826 \mathrm{mg}$ ) which was separated by a reverse-phase Lichroprep Lobar RP-18 column (size B, $310 \times$ $25 \mathrm{~mm}$; Merck, Germany), eluted with $\mathrm{MeOH}-$ formic acid ( $0.1 \%$, aqueous solution) (3:7), flow rate $2 \mathrm{ml} / \mathrm{min}$, to give five fractions (E4-D1 5). Fraction E4-D2 (337 mg) was proanthocyanin A1 (2-nor-ent) [9]. Part of fraction E4-D4 (36 $\mathrm{mg}$ out of $360 \mathrm{mg}$ ) was separated on a semi-preparative HPLC column (Phenomenex ${ }^{\circledR}$ Prodigy 100A ODS3, $250 \times 10 \mathrm{~mm}, 5$ $\mu \mathrm{m}$; Phenomenex, Torrance, California, USA) $(12 \times 3 \mathrm{mg})$, eluted with $\mathrm{MeOH}$-formic acid (0.1\%, aqueous solution) (1:3) at $35^{\circ} \mathrm{C}$, flow rate $2 \mathrm{ml} / \mathrm{min}$, to give epicatechin- $(2 \beta \rightarrow O \rightarrow 7$, $4 \beta \rightarrow 8)$-ent-epicatechin (3-nor-ent) $\left(9.9 \mathrm{mg}, t_{\mathrm{R}} 23.12 \mathrm{~min}\right)$ and proanthocyanidin A2 (3-nor) $\left(6.0 \mathrm{mg}, t_{\mathrm{R}} 29.44 \mathrm{~min}\right)$ [9].

An aliquot of fraction E7 (991 mg out of $1.87 \mathrm{~g}$ ) was fractionated using the same CPC condition as that for fraction E4 to give three fractions [E7-A1 3; mobile phase: lower layer (E7-A1 2), upper layer (E7-A3)]. Fraction E7-A2 (120 mg) was separated by RP-18 Lobar column (size A, $240 \times 10$ $\mathrm{mm}$; Merck, Germany), eluted with $25 \sim 30 \% \mathrm{MeOH}-$ acetic acid $(0.1 \%$, aqueous solution) $(3: 7)$, flow rate $1 \mathrm{ml} / \mathrm{min}$, to give six fractions (E7-A2-1 6). Fraction E7-A2-3 was epicatchin- $(2 \beta \rightarrow O \rightarrow 7,4 \beta \rightarrow 6)$-catechin (Iso-2-nor-ent, 9.4 mg) [9].

\section{3. a-Glucosidase Inhibition Assay}

Test compounds (2-nor-ent, 3-nor, 3-nor-ent, and iso-2-nor-ent) were dissolved initially in $10 \% \mathrm{MeOH}$, whose final concentration was $1 \%$ while measuring the inhibitory activity against $\alpha$-glucosidase (Sigma-Aldrich G3651, Enzyme Commission (EC) number 3.2.1.20, GenBank: BAA12704.1, Sigma-Aldrich Co., Germany), following the reported procedure [8]. The $\mathrm{IC}_{50}$ values were calculated by GraphPad Prism 5 software from three experiments. The positive control Acarbose (Bayer) was found to have an $\mathrm{IC}_{50}$ value of $9.71 \mathrm{nM}$ against the same enzyme.

\section{Results and Discussion}

\subsection{Docking Calculation}

An inhibitory compound with a high binding energy (i.e. bigger negative numbers) should have a low $\mathrm{IC}_{50}$. Molecular docking of compounds $1-8$ and the de-unit III homologs of $2-8$ on $\alpha$-glucosidase was undertaken initially via London (trimatch)-refinement (Forcefield-Affinity $\Delta G$ and Forcefield-London) mode and the results were listed in Tables 
1 and 2 . The correlation between docking score and bioassay result $\left(\mathrm{IC}_{50}\right)$ is roughly consistent. However, the binding scores yielded from Forcefield-Affinity $\Delta G$ mode agreed better with bioassay results $\left(\mathrm{IC}_{50}\right)$. Further docking and bioassay study on four proanthocyanidins (2-nor-ent, 3-nor, 3-nor-ent, and iso-2-nor-ent), isolated from peanut skins [9], also confirmed this observation. As such, molecular docking of 13 isomeric trimeric proanthocyanidin isomers at the C-2 and $\mathrm{C}-3$ positions in units II and III on $\alpha$-glucosidase was undertaken via London (trimatch)-refinement (Forcefield-Affinity $\Delta G$ ) mode. The result was shown in Table 3.

\subsubsection{Interaction of AG with Proanthocyanidins from Machilus Philippinesis}

Our investigation was primarily based on the interplay of conformational change and spatial orientation associated with the binding affinity. Acarbose showed six H-bonds and five ionic interactions with the constructed docking model of $\alpha$-glucosidase (EC 3.2.1.20 from Bacillus stearothermophilus) [7]. As listed in Table 1, the calculated docking energies were in good accordance with the $\mathrm{IC}_{50}$ values.

As shown, while the binding affinity increased, the IC50 value decreased. Among these, the trimeric aesculitannin B (2) fitted into the hydrophobic pocket of AG with hydrophobic interactions, which strongly associated with F649, and hydrogen-bonding interactions with the side chain of D443 (Figure 3A; Figure A1, Supplementary data). It was noted that the $7-\mathrm{OH}$ in unit I had preferred orientation towards the binding pocket interior. These interactions made 2 the most potent against $\mathrm{AG} \quad\left(\mathrm{IC}_{50} \quad 3.5 \mu \mathrm{M}\right)$ among these proanthocyanidins. As for the other two trimeric isomers (3 and 4), they showed much weaker anti-AG activity, exemplified by their low docking affinity to AG (Table 1).

a

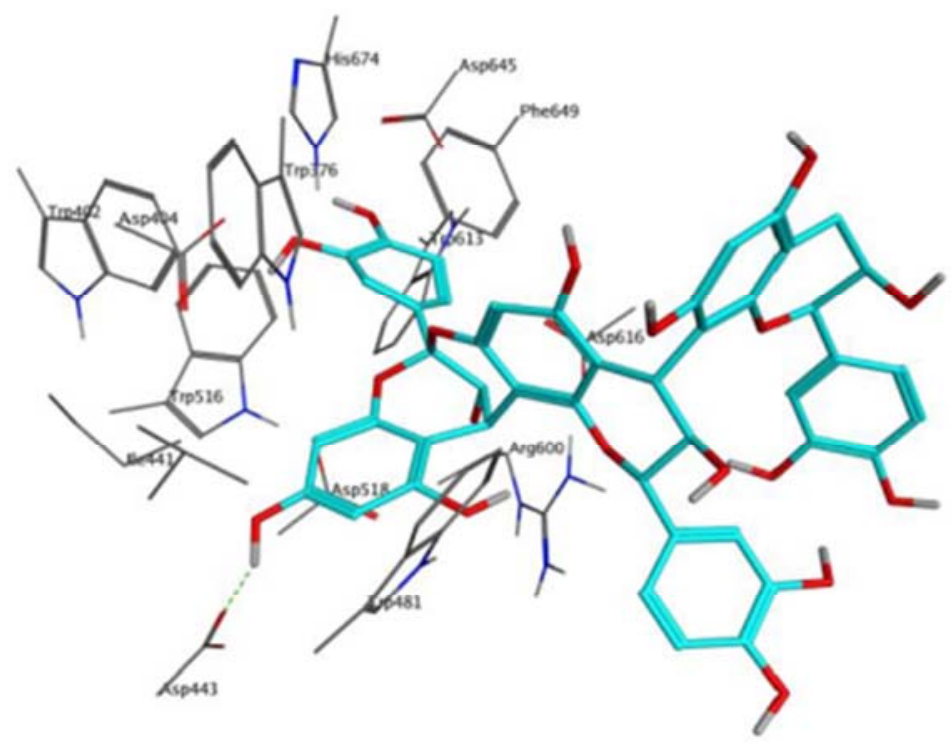

b

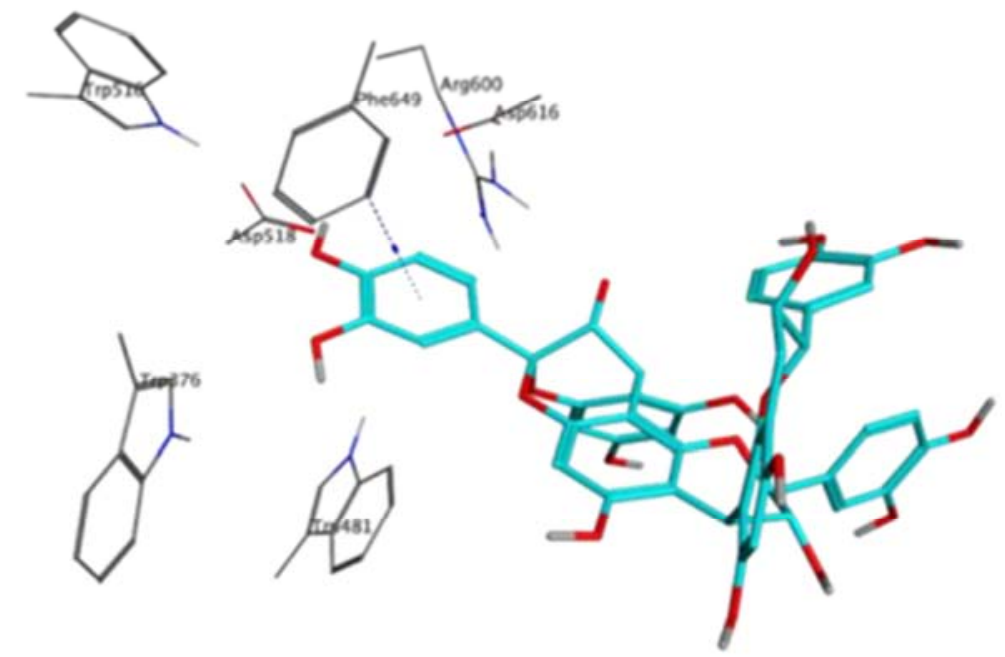




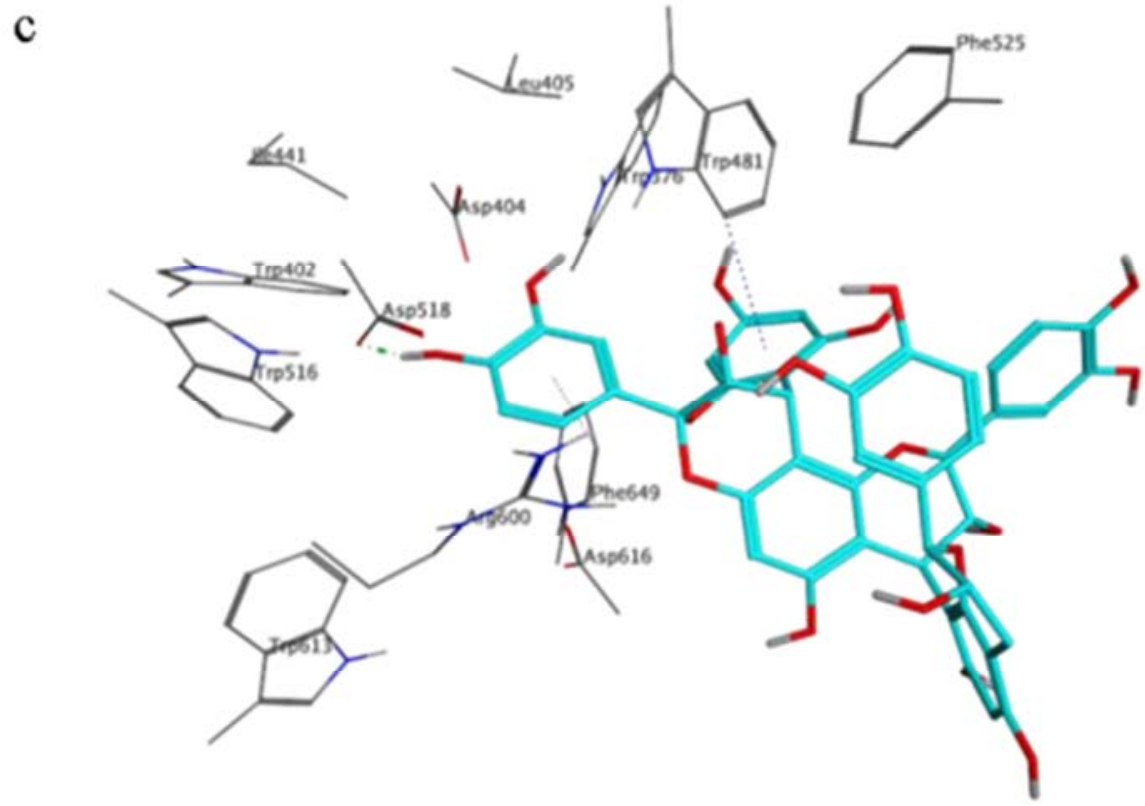

Figure 3. Molecular docking of trimeric proanthocyanidins 2 (aesculitannin B) (a), 2-entC (b), and 2-C (c) to the constructed a-glucosidase.

For the tetrameric isomers, pavetannin C-1 (5), having an additional epicatechin residue $(6 \rightarrow 4 \beta)$-linked to unit $\mathrm{I}$ of cinnamtannin B-1 (3), is more potent than parameritannin A-1 (6), having an additional epicatechin residue $(6 \rightarrow 4 \beta)$-linked to unit II of 3 . The better docking affinity of 5 than 6 to the active site $(\Delta G-20.46$ vs. -8.01$)$ could be explained by possessing two more H-bonds (unit I' $7-\mathrm{OH}$ and 4'-OH to D443 and D645, respectively) (Figure 4A; Figure A2, Supplementary data). The additional epicatechin moiety in 6 was observed to hamper the flexibility of unit III, leading to a poor docking score to AG (Figure 4A; Figure A2A,
Supplementary data). Machiphilitannin A (7), having an additional epicatechin residue $(8 \rightarrow 4 \beta)$-linked to unit II' of 6 , had better structure flexibility than 6 , arisen from the additional moiety. This allowed more $n-\pi$ interactions to $A G$ and made 7 have better anti-AG activity than $6\left(\mathrm{IC}_{50} 31.3 \mu \mathrm{M}\right.$ vs. $>100 \mu \mathrm{M}$ ). The structure of machiphilitannin B (8), having an additional epicatechin residue $(8 \rightarrow 4 \beta)$-linked to II" of 7 , was even more flexible than that of 7 , allowing 8 to have one H-bond more than 7, thus had better anti-AG activity ( $\mathrm{IC}_{50} 18.4 \mu \mathrm{M}$ vs. $\left.31.3 \mu \mathrm{M}\right)$ (Table 1$)$.

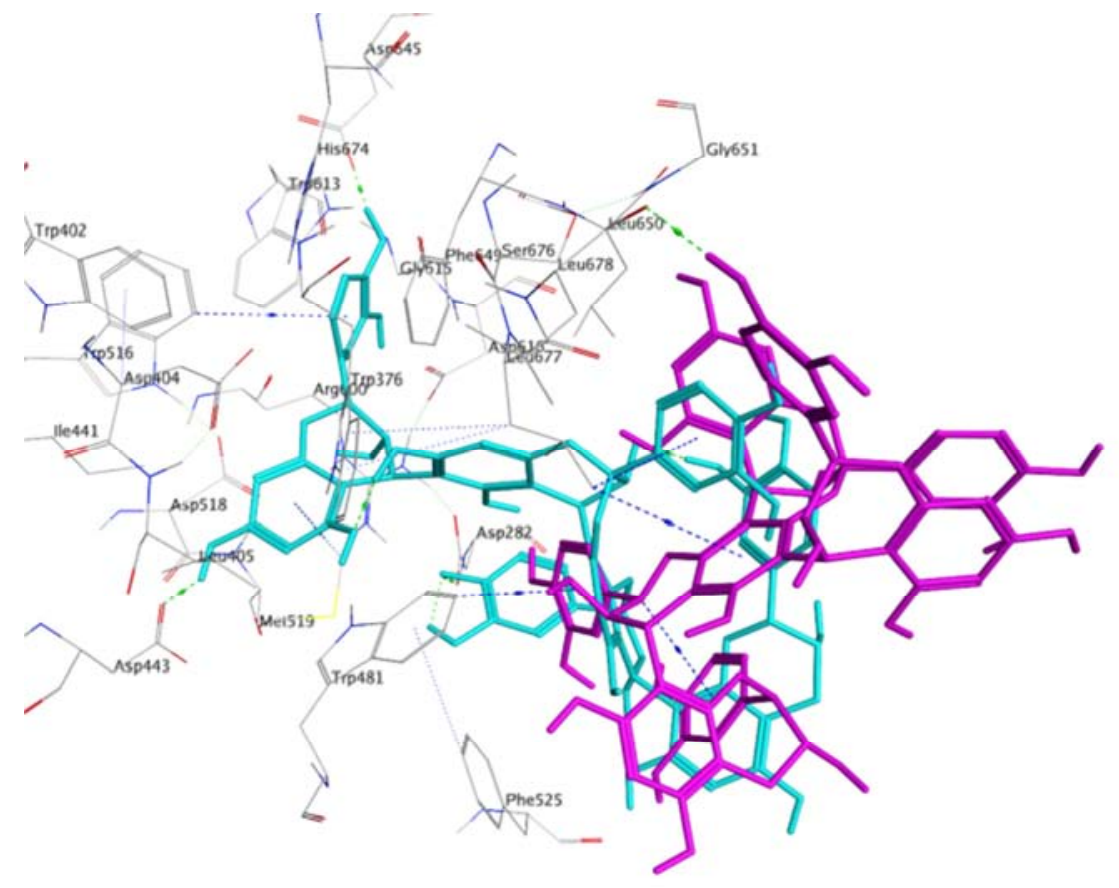

(a) 


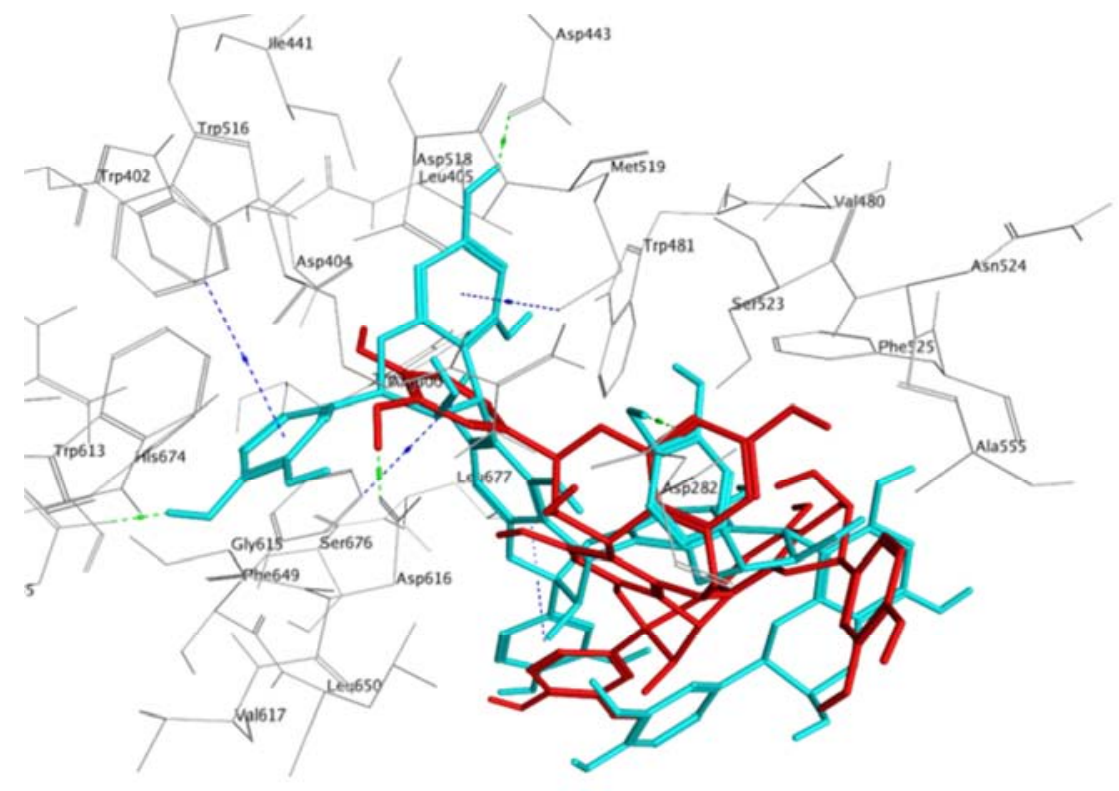

(b)

Figure 4. Molecular docking of oligo proanthocyanidins 5 (pavetannin C-1) versus 6 (parameritannin A-1) (a: 5, light blue; 6, purple), and 5 versus 5-nor (b: 5 , light blue; 5-nor, red) to the constructed $\alpha$-glucosidase model.

\subsubsection{Interaction of AG with de-unit III Homologues of $2-8$}

To examine the contribution of unit III to the anti-AG activity, this unit in $2-8$ was truncated to give nor $2-8$. Their virtual binding affinity to AG was shown in Table 2 .

Table 1. Correlation of $I C_{50}$ and molecular docking score $(\Delta G)$ of eight proanthocyanidins (1-8), isolated from Machilus philippinensis, toward $\alpha$-glucosidase.

\begin{tabular}{|c|c|c|c|c|c|c|c|c|c|c|}
\hline \multirow{3}{*}{$\operatorname{Compd}^{a}$} & \multirow{3}{*}{$\mathrm{IC}_{50}(\mu \mathrm{M})^{9}$} & \multirow{3}{*}{$\Delta G_{\text {calc }}{ }^{*}, b(\mathrm{kcal} / \mathrm{mol})$} & \multicolumn{8}{|c|}{ Number of Ligand-Receptor Interaction ${ }^{b}$} \\
\hline & & & \multicolumn{2}{|c|}{ H-bond } & \multicolumn{2}{|l|}{$n-\pi$} & \multicolumn{2}{|c|}{$\pi-\pi$} & \multicolumn{2}{|c|}{ ionic } \\
\hline & & & $\mathbf{T}^{c}$ & $\mathbf{A}^{d}$ & $\mathbf{T}$ & $\mathbf{A}$ & $\mathbf{T}$ & $\mathbf{A}$ & $\mathbf{T}$ & $\mathbf{A}$ \\
\hline Monomeric 1 & $>100.0$ & $-7.87 /-10.74$ & $1 / 2$ & $0 / 2$ & $1 / 0$ & $1 / 0$ & $0 / 0$ & $0 / 0$ & $0 / 0$ & $0 / 0$ \\
\hline Trimeric 2 & 3.5 & $-21.48 /-20.62$ & $2 / 5$ & $1 / 4$ & $1 / 0$ & $0 / 0$ & $1 / 0$ & $1 / 0$ & $1 / 0$ & $1 / 0$ \\
\hline 3 & $>100.0$ & $-6.43 /-9.92$ & $0 / 1$ & $0 / 0$ & $1 / 2$ & $1 / 1$ & $0 / 0$ & $0 / 0$ & $0 / 0$ & $0 / 0$ \\
\hline 4 & 92.9 & $-8.17 /-11.54$ & $0 / 3$ & $0 / 2$ & $1 / 2$ & $1 / 2$ & $0 / 0$ & $0 / 0$ & $0 / 0$ & $0 / 0$ \\
\hline Tetrameric 5 & 10.5 & $-20.46 /-16.41$ & $3 / 1$ & $2 / 0$ & $2 / 3$ & $2 / 2$ & $1 / 0$ & $1 / 0$ & $0 / 0$ & $0 / 0$ \\
\hline Pentameric 7 & 31.3 & $-12.77 /-12.55$ & $1 / 2$ & $0 / 1$ & $4 / 2$ & $4 / 2$ & $0 / 0$ & $0 / 0$ & $0 / 0$ & $0 / 0$ \\
\hline Hexameric 8 & 18.4 & $-15.48 /-15.45$ & $2 / 2$ & $1 / 1$ & $3 / 3$ & $3 / 1$ & $0 / 0$ & $0 / 0$ & $0 / 0$ & $0 / 0$ \\
\hline acarbose & 0.049 & $-23.21 /-20.66$ & $6 / 4$ & $3 / 2$ & $1 / 0$ & $1 / 0$ & $0 / 0$ & $0 / 0$ & $5 / 3$ & $4 / 2$ \\
\hline
\end{tabular}

${ }^{a}$ Epicatechin (1), aesculitannin B (2), cinnamtannin B-1 (3), cinnamtannin D-1 (4), pavetannin C-1 (5), parameritannin A-1 (6), machiphilitannin A (7), machiphilitannin B (8); ${ }^{*}$ Free binding energy $(\Delta G, \mathrm{kcal} / \mathrm{mol}) ;{ }^{b}$ refinement: Forcefield- Affinity $\Delta G /$ Forcefield- London; ${ }^{c}$ total interaction number; ${ }^{d}$ interaction number at the active site.

Table 2. Molecular docking score $(\Delta G)$ of de-unit III of $\mathbf{2}-\mathbf{8}\left(\mathbf{2}-\mathbf{8}\right.$ nor) and $I_{50}$ of four dimeric A-type proanthocyanidins toward $\alpha$-glucosidase.

\begin{tabular}{|c|c|c|c|c|c|c|c|c|c|c|}
\hline \multirow{3}{*}{$\operatorname{Compd}^{a}$} & \multirow{3}{*}{$\mathrm{IC}_{50}(\mu \mathrm{M})$} & \multirow{3}{*}{$\begin{array}{l}\Delta G_{\text {calc }}^{*, b} \\
(\mathrm{kcal} / \mathrm{mol})\end{array}$} & \multicolumn{8}{|c|}{ Number of Ligand-Receptor Interaction ${ }^{b}$} \\
\hline & & & \multicolumn{2}{|c|}{ H-bond } & \multicolumn{2}{|l|}{$n-\pi$} & \multicolumn{2}{|l|}{$\pi-\pi$} & \multicolumn{2}{|c|}{ ionic } \\
\hline & & & $\mathbf{T}^{c}$ & $\mathrm{~A}^{d}$ & $\mathbf{T}$ & $\mathbf{A}$ & $\mathbf{T}$ & $\mathbf{A}$ & $\mathbf{T}$ & $\mathbf{A}$ \\
\hline Dimeric 2-nor & & $-16.66 /-16.67$ & $2 / 2$ & $1 / 1$ & $1 / 1$ & $0 / 0$ & $1 / 1$ & $1 / 1$ & $0 / 0$ & $0 / 0$ \\
\hline 2-nor-ent & 96.3 & $-16.85 /-16.81$ & $2 / 2$ & $1 / 1$ & $1 / 2$ & $0 / 1$ & $0 / 1$ & $0 / 1$ & $0 / 0$ & $0 / 0$ \\
\hline 3-nor (=4-nor) & 86.4 & $-16.51 /-16.13$ & $2 / 2$ & $2 / 2$ & $1 / 2$ & $0 / 2$ & $0 / 1$ & $0 / 1$ & $0 / 0$ & $0 / 0$ \\
\hline 3-nor-ent & 155.3 & $-14.72 /-14.71$ & $0 / 0$ & $0 / 0$ & $1 / 1$ & $0 / 0$ & $1 / 1$ & $1 / 1$ & $0 / 0$ & $0 / 0$ \\
\hline Iso-2-nor-ent & 9.7 & $-19.13 /-17.76$ & $4 / 2$ & $3 / 2$ & $2 / 1$ & $1 / 1$ & $1 / 1$ & $1 / 1$ & $0 / 1$ & $0 / 1$ \\
\hline 6-nor & & $-11.11 /-10.98$ & $2 / 1$ & $1 / 1$ & $1 / 1$ & $1 / 1$ & $0 / 1$ & $0 / 1$ & $0 / 0$ & $0 / 0$ \\
\hline Tetrameric 7-nor & & $-13.83 /-12.03$ & $1 / 1$ & $1 / 1$ & $1 / 1$ & $0 / 0$ & $0 / 0$ & $0 / 0$ & $0 / 0$ & $0 / 0$ \\
\hline Pentameric 8-nor & & $-16.55 / 16.55$ & $2 / 2$ & $1 / 1$ & $2 / 2$ & $0 / 0$ & $0 / 0$ & $0 / 0$ & $0 / 0$ & $0 / 0$ \\
\hline acarbose & 0.023 & $-23.21 /-20.66$ & $6 / 4$ & $3 / 2$ & $1 / 0$ & $1 / 0$ & $0 / 0$ & $0 / 0$ & $5 / 3$ & $4 / 2$ \\
\hline
\end{tabular}

${ }^{*}$ Free binding energy $(\Delta G, \mathrm{kcal} / \mathrm{mol})$; ${ }^{a}$ 2-nor-ent: proanthocyanidin $\mathrm{A} 1$, unit II enantiomer of 2-nor; 3-nor: proanthocyanidin A2; 3-nor-ent:

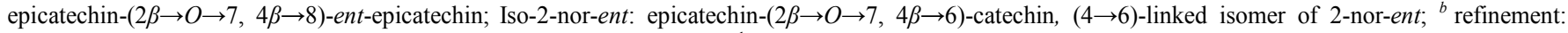
Forcefield- Affinity $\Delta G /$ Forcefield- London; ${ }^{c}$ total interaction number; ${ }^{d}$ interaction number at the active site 


\subsubsection{Correlation of $I_{50}$ and Docking Score to AG of Four Dimeric Proanthocyanidins (2-nor-ent, iso-2-nor-ent, 3-nor, and 3-nor-ent)}

Among the dimeric compounds for this docking study, epicatechin- $(2 \beta \rightarrow O \rightarrow 7,4 \beta \rightarrow 8)$-ent-epi-catechin (2-nor-ent), proanthocyanidin A2 (3-nor), proanthocyanin A1 (3-nor-ent), and epicatechin- $(2 \beta \rightarrow O \rightarrow 7,4 \beta \rightarrow 6)$-catechin (iso-2-nor-ent) had been isolated from peanut skin [9] but not readily available due to tough and tedious isolation steps. To verify their virtual screening result, they were re-isolated in this study. The bioassay result was shown in Table 2. Despite some quantitative discrepancies were observed between the correlation of $\mathrm{IC}_{50}$ values and docking scores for these dimeric isomers while comparing to those correlations of 2-8, they still presented a qualitative consistency.

According to the binding affinity and the orientations of the dimeric isomers (2-nor-ent and 3-nor) to $\mathrm{AG}$, the unit II 4 '-OH, potentially responsible for hydrogen bonding to $A G$, was oriented toward the binding pocket interior. The higher binding affinity of 3-nor than that of 3 was due to the smaller molecular size, making it readily accessible into binding pocket. Since 2-nor and 2-nor-ent constituted more compact conformations than 2, they cannot form an ionic interaction with $\mathrm{AG}$ as that of 2, leading to much weaker binding affinity.

To validate our docking calculations, the same docking approach was carried out for epicatechin- $(2 \beta \rightarrow O \rightarrow 7$, $4 \beta \rightarrow 6$ )-catechin (iso-2-nor-ent) [9]. Its docking score value was well correlated with $\mathrm{IC}_{50}$ value (Table 2). While comparing to the isomer 2-nor-ent, iso-2-nor-ent showed much better docking score. Such difference could be rationalized as follows. The steric hindrance effect derived from the $4 \beta \rightarrow 8$ linkage in 2-nor-ent, which influenced the spatial orientation and shifted its unit II residue toward the binding pocket interior of $\mathrm{AG}$, forming only one $\mathrm{H}$-bond (3'-OH to H674) (Figure 5). As for iso-2-nor-ent, its unit I residue migrated toward the binding pocket interior to form three H-bonds (5-OH to $\mathrm{W} 481,7-\mathrm{OH}$ to $\mathrm{D} 404$, and 3'-OH to D645), one $\pi-\pi$ and one $n-\pi$ interactions with AG (Figure 5).

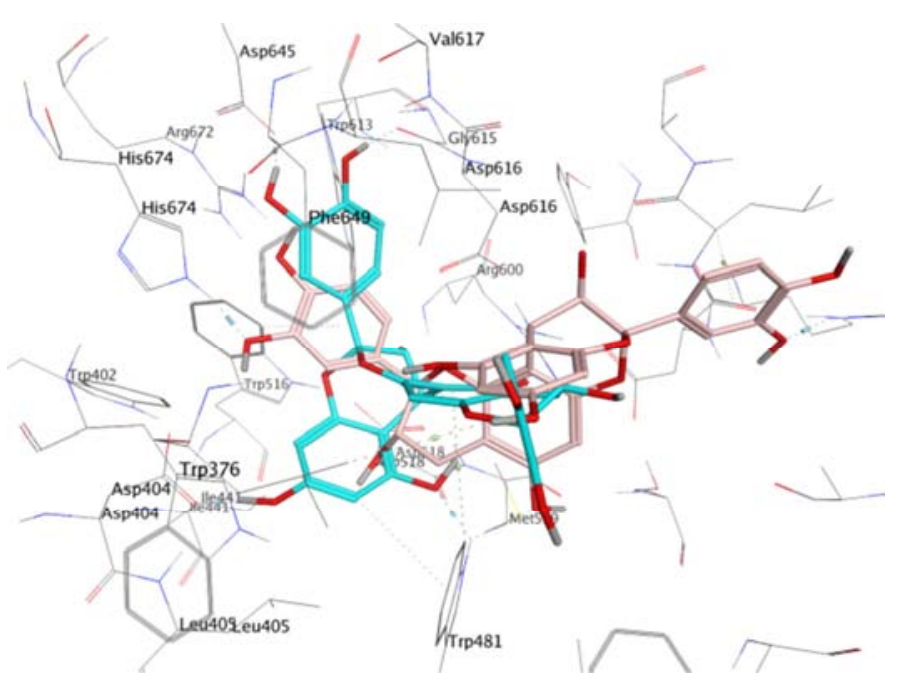

(a)

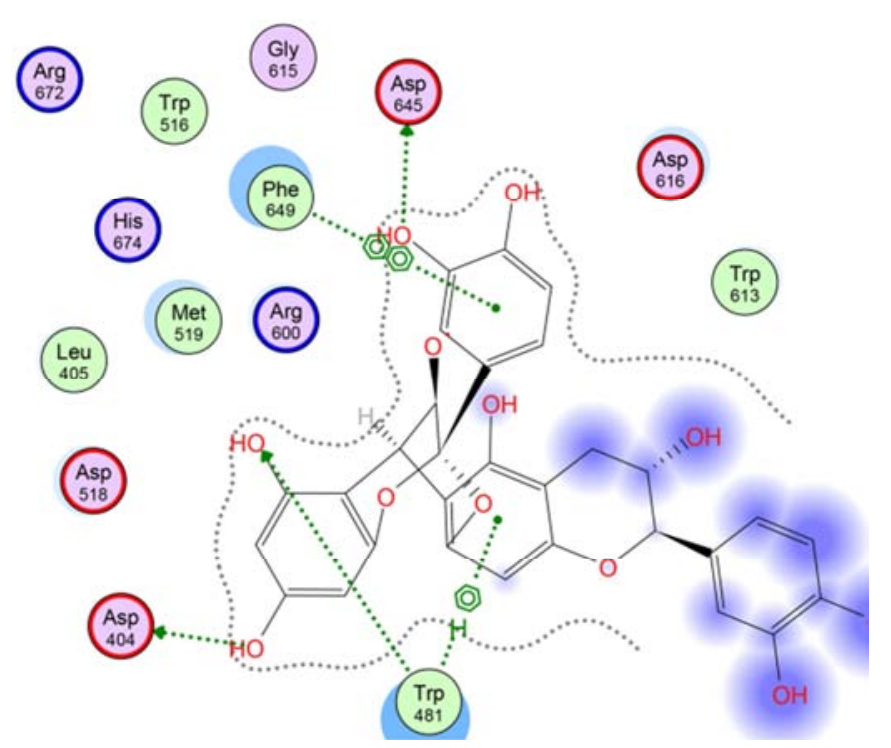

(b) 


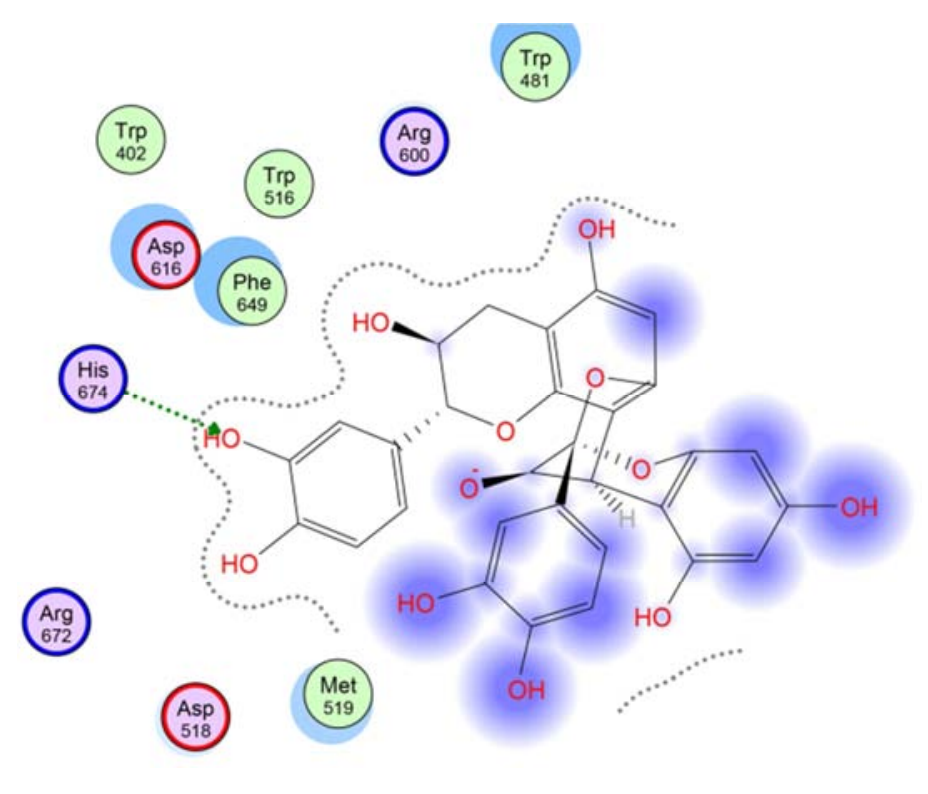

(c)

Figure 5. Molecular docking of 2-nor-ent (a, pink) versus iso-2-nor-ent (a, light blue), iso-2-nor-ent (b), and 2-nor-ent (c) to the constructed $\alpha$-glucosidase model

\subsubsection{Correlation of $I C_{50}$ and Docking Score to $A G$ of De-unit III Homologues of 5-8}

As indicated above, the steric hindrance between unit III and unit II' will reduce the flexibility and hence decrease binding affinity. Thus truncation of this unit from 6-8 might increase the binding affinity. This is the case since the nor-analogues of 6-8 showed better docking scores than the corresponding parent compounds (Tables 1 and 2).

Removal of unit III from 5 (5-nor), however, decreased the binding affinity ascribable to the orientation change while docking as shown in Figure 4B and Figure A2B (Supplementary data). Thus, the spatial orientations of the flavan-3-ol residue should play an important role in designing AG inhibitors. It was noted that the phenolic hydroxyl groups not only served as the source of hydrogen bonding but also contributed lone-pair electrons to enhance $n-\pi$ interactions to $\mathrm{AG}$, providing additional stability to these complexes.

\subsubsection{Interaction of AG with Trimeric Proanthocyanidins of A-type Interflavanoids}

As the trimeric aesculitannin B (2) has much stronger anti- $\alpha$-glucosidase activity than the isomeric cinnamtannins B1 (3) and D-1 (4) (Table 1), the configuration at C-2 and $\mathrm{C}-3$ in unit II seems play a key role. A comprehensive docking study on the trimeric compounds with various configuration at C-2 and C-3 of units II and III (Figure 2), consisting of epicatechin $(2 R, 3 R)(\mathrm{E})$, catechin $(2 R, 3 S)(\mathrm{C})$, ent-epicate-chin $(2 S, 3 S)($ entE), and ent-catechin $(2 S, 3 R)$ $(e n t C)$, was performed to verify their roles in this bioactivity. The result was shown in Table 3. Among the compounds for this docking study, two trimeric compounds (3C and 2-ent $\mathrm{C}$ ) have been isolated from the nature $[17,18]$.

\subsubsection{Interaction of $A G$ with 3-entC, 3C-entE, 3ent-C, and 2}

As indicated, those with unit II identical to unit III, e.g. 3 (both units being epicatechin), showed almost the worst docking affinity. While 3-ent $\mathrm{C}, 3 \mathrm{C}$-ent $\mathrm{E}, 3$ ent-C, and 2 possessing the same chirality at $\mathrm{C}-3$ in both units but different at $\mathrm{C}-2$, e.g. 3-ent $\mathrm{C}$ having $3-R$ in both units, 2-S in unit II but $2-R$ in unit III, showed good docking score. As shown, the structure conformations of 2 (Figure 3A; Figure A1, Supplementary data) and 3-entC (Figure A3, Supplementary data) were folded into an extended and flexible shape, allowing them to have better docking probability.

\subsubsection{Interaction of $A G$ with 2-entE, 3ent-entC, 3ent-entE, and 2-entC}

It was noted that if both units are enantiomers of catechin or epicatechin, i.e. ent-C or ent-E, the composed compounds, 2-ent $\mathrm{E}$, 3ent-ent $\mathrm{C}$, 3ent-ent $\mathrm{E}$, and 2-ent $\mathrm{C}$, showed poor docking score. 2-ent $\mathrm{C}$, an epimer of 2 at the unit III C-2 position, lacks the ionic interaction and hydrogen bonding with AG active site relative to 2 (Table 3, Figure 3B), rationalizing its poor docking score.

\subsubsection{Interaction of $A G$ with $3 C$, 3ent, 2-C, 4, 3-entE, and $3 C$-entC}

$3 \mathrm{C}$ and $3 e n t$, both possessing $3 S$ - at unit II and $2 R$ - at unit III, showed good docking score. Compound 2-C, a C-3 epimer at unit III of 2 , showed a weaker docking score than $2(\Delta G-16.68$ vs. $-21.48 \mathrm{Kcal} / \mathrm{mole}$ ) by lacking the ionic interaction with $\mathrm{AG}$ relative to 2 (Figure $3 \mathrm{C}$ ).

As those of 2 and 3-ent $\mathrm{C}$, the structures of $3 \mathrm{C}$, 3ent, and 2-C were also folded into an extended and flexible shape (Figure A4, Supplementary data) and thus have better docking. While compounds $4(3-\mathrm{C}), 3-e n t \mathrm{E}$, and $3 \mathrm{C}$-ent $\mathrm{C}$ with the corresponding exchanged units II and III moieties as those three latter mentioned compounds (3C, 3ent, and 2-C) folded into compact and fairly rigid structures due to intramolecular hydrogen bonding or/ and $\pi-\pi$ interaction (for 4: Figure A5, 
Supplementary data), leading to much weaker docking capacity.

The visual comparison of the MD simulation of these trimeric ligands to AG confirmed that the binding site of $A G$ preferred to adopt a rather extended conformation instead of a compact one.

With the observation of various complex orientations, the MD simulation showed that residues D404, D443. D518, D524, and D616 appeared to be responsible for potentially favorable hydrogen-bonding interactions between the oligomeric 5,7,3',4'-tetrahydroxyflavan-3-ols and the AG active site, similar to those reported for the active-site pocket (D404, I441, W481, W516, D518, M519, R600, D616, F649, and H674) [19] and those responsible for key catalytic activity (D518 and D616) [20-22]. As shown in Table 3, $\pi-\pi$ interactions, mainly arisen from ring $\mathrm{B}$ of unit $\mathrm{I}$ in the ligands with the phenylalanine residue in AG, are also responsible for some bonding strength. The present study also indicated that for the good docking compounds such as 2 in Tables 1 and 3, the oxygen atom of the $3-\mathrm{OH}$ in unit I formed an ionic bond with the guanidinium nitrogen of A600 in AG (Figure 3A), greatly increasing the docking score.

\section{Conclusion}

In this work we have demonstrated a good correlation between the $\mathrm{IC}_{50}$ values against $\alpha$-glucosidase and in silico molecular docking scores using our built $\alpha$-glucosidase model on seven proanthocyanidins, isolated from Machilus philippinensis (2-8). Extension of this study was undertaken on eight de-unit III homologs of $2-8$, and 13 trimeric proanthocyanidin, belonging to stereoisomers of 2 at C-2 and C-3 of units II and III. The simulation profiles of binding energy displayed a reasonable qualitative agreement with the $\mathrm{IC}_{50}$ on the dimeric 2-nor-ent, 3-nor, 3-nor-ent, and iso-2-nor-ent, isolated from peanut skins, partially verifying this MD study. In addition, five trimeric isomers (3-ent $\mathrm{C}$, $3 \mathrm{C}$-ent $\mathrm{E}$, 3ent- $\mathrm{C}, 3 \mathrm{C}$, and 3ent) were demonstrated to have docking score comparable to aesculitannin $\mathrm{B}\left(2, \mathrm{IC}_{50} 3.5 \mu \mathrm{M}\right)$ and they should be of value for further exploration as $\alpha$-glucosidase inhibitors.

Table 3. Interaction between $\alpha$-glucosidase and trimeric proanthocyanidin isomers at the C-2 and C-3 positions in units II and III, based on molecular docking.

\begin{tabular}{|c|c|c|c|c|c|c|c|c|c|c|c|c|c|c|c|c|c|}
\hline \multirow{3}{*}{$\operatorname{Compd}^{a}$} & \multirow{3}{*}{ Unit II } & \multirow{2}{*}{\multicolumn{2}{|c|}{ Configuration }} & \multirow{3}{*}{ Unit III } & \multirow{2}{*}{\multicolumn{2}{|c|}{ Configuration }} & \multirow{2}{*}{\multicolumn{2}{|c|}{$\begin{array}{l}\text { Configuration } \\
\text { Unit II vs. III }\end{array}$}} & \multirow{3}{*}{$\Delta \boldsymbol{G}_{\text {calc }}{ }^{*, b}$} & \multicolumn{8}{|c|}{ Number of Ligand-Receptor Interaction } \\
\hline & & & & & & & & & & \multicolumn{2}{|c|}{ H-bond } & \multicolumn{2}{|c|}{$n-\pi$} & \multicolumn{2}{|c|}{$\pi-\pi$} & \multicolumn{2}{|c|}{ Ionic } \\
\hline & & $\mathrm{C}-2$ & $\mathrm{C}-3$ & & $\mathrm{C}-2$ & C-3 & $\mathrm{C}-2$ & C-3 & & $\mathbf{T}^{c}$ & $\mathrm{~A}^{d}$ & $\mathbf{T}$ & $\mathbf{A}$ & $\mathbf{T}$ & $\mathbf{A}$ & $\mathbf{T}$ & $\mathbf{A}$ \\
\hline 3-ent $\mathrm{C}$ & epicat & $\mathrm{R}$ & $\mathrm{R}$ & ent-cat & $\mathrm{S}$ & $\mathrm{R}$ & $\operatorname{diff}^{\mathrm{e}}$ & same & -21.72 & 2 & 1 & 1 & 0 & 1 & 1 & 1 & 1 \\
\hline $3 \mathrm{C}-e n t \mathrm{E}$ & cat & $\mathrm{R}$ & $\mathrm{S}$ & ent-epic & $\mathrm{S}$ & $\mathrm{S}$ & diff. & same & -19.39 & 2 & 1 & 0 & 0 & 1 & 1 & 1 & 1 \\
\hline 3ent $-\mathrm{C}$ & ent-epic & $\mathrm{S}$ & $\mathrm{S}$ & cat & $\mathrm{R}$ & $\mathrm{S}$ & diff. & same & -19.53 & 1 & 1 & 3 & 2 & 1 & 1 & 2 & 1 \\
\hline 2 & ent-cat & $\mathrm{S}$ & $\mathrm{R}$ & epicat & $\mathrm{R}$ & $\mathrm{R}$ & diff. & same & -21.48 & 2 & 1 & 1 & 0 & 1 & 1 & 1 & 1 \\
\hline $3 \mathrm{C}-\mathrm{C}$ & cat & $\mathrm{R}$ & $\mathrm{S}$ & cat & $\mathrm{R}$ & $\mathrm{S}$ & same & same & -7.08 & 1 & 0 & 1 & 1 & 0 & 0 & 0 & 0 \\
\hline 3ent-ent $\mathrm{E}$ & ent-epic & $\mathrm{S}$ & $\mathrm{S}$ & ent-epic & $\mathrm{S}$ & $\mathrm{S}$ & same & same & -8.93 & 0 & 0 & 1 & 1 & 0 & 0 & 0 & 0 \\
\hline 2-entC & ent-cat & $\mathrm{S}$ & $\mathrm{R}$ & ent-cat & $\mathrm{S}$ & $\mathrm{R}$ & same & same & -8.12 & 0 & 0 & 1 & 1 & 0 & 0 & 0 & 0 \\
\hline $3 \mathrm{C}$ & cat & $\mathrm{R}$ & $\mathrm{S}$ & epicat & $\mathrm{R}$ & $\mathrm{R}$ & same & diff. & -20.09 & 4 & 2 & 0 & 0 & 1 & 1 & 1 & 1 \\
\hline $3 e n t$ & ent-epic & $\mathrm{S}$ & $\mathrm{S}$ & epicat & $\mathrm{R}$ & $\mathrm{R}$ & diff. & diff. & -19.73 & 2 & 2 & 1 & 0 & 1 & 1 & 1 & 1 \\
\hline $3 \mathrm{C}-e n t \mathrm{C}$ & cat & $\mathrm{R}$ & $\mathrm{S}$ & ent-cat & $\mathrm{S}$ & $\mathrm{R}$ & diff. & diff. & -8.33 & 0 & 0 & 1 & 1 & 0 & 0 & 0 & 0 \\
\hline $4(=3-C)$ & epicat & $\mathrm{R}$ & $\mathrm{R}$ & cat & $\mathrm{R}$ & $\mathrm{S}$ & same & diff. & -8.17 & 0 & 0 & 1 & 1 & 0 & 0 & 0 & 0 \\
\hline 2-ent $\mathrm{E}$ & ent-cat & $\mathrm{S}$ & $\mathrm{R}$ & ent-epic & $\mathrm{S}$ & $\mathrm{S}$ & same & diff. & -7.64 & 0 & 0 & 1 & 1 & 0 & 0 & 0 & 0 \\
\hline 3ent-ent $\mathrm{C}$ & ent-epic & $\mathrm{S}$ & $\mathrm{S}$ & ent-cat & $\mathrm{S}$ & $\mathrm{R}$ & same & diff. & -7.46 & 0 & 0 & 1 & 1 & 0 & 0 & 0 & 0 \\
\hline $2-\mathrm{C}$ & ent-cat & $\mathrm{S}$ & $\mathrm{R}$ & cat & $\mathrm{R}$ & $\mathrm{S}$ & diff. & diff. & -16.68 & 2 & 1 & 2 & 1 & 0 & 0 & 0 & 0 \\
\hline
\end{tabular}

${ }^{*}$ Free binding energy $(\Delta G, \mathrm{kcal} / \mathrm{mol}) ;{ }^{a}$ ent: enantiomer, $C$ : catechin (cat), E: epicatchin (epic), 3-entC: an isomer of 3 with unit III as ent-catechin, $3 C$-entE: an isomer of 3 with unit II as catechin and unit III as ent-epicatechin; ${ }^{b}$ refinement: Forcefield- Affinity $\Delta G$; ${ }^{c}$ total interaction number; ${ }^{d}$ interaction number at active site; ${ }^{e}$ different configuration.

First and foremost, the docking analysis is very useful in virtual screening. While examining the docking data, not only the scores are concerned but also the conformation of ligand fitted to the target (AG in this study) since some better scores might come from unlikely twisted conformation. Our study has provided encouraging idea for screening in selecting compounds for further investigation.

\section{Acknowledgements}

We gratefully acknowledge the supply of peanut skins from Today peanut food processing plant, Xiluo, Yunlin County, Taiwan, and the financial support from Ministry of Science and Technology, Taiwan, ROC under the grant NSC 97-2320-B-002-012-MY3. 


\section{Appendix}
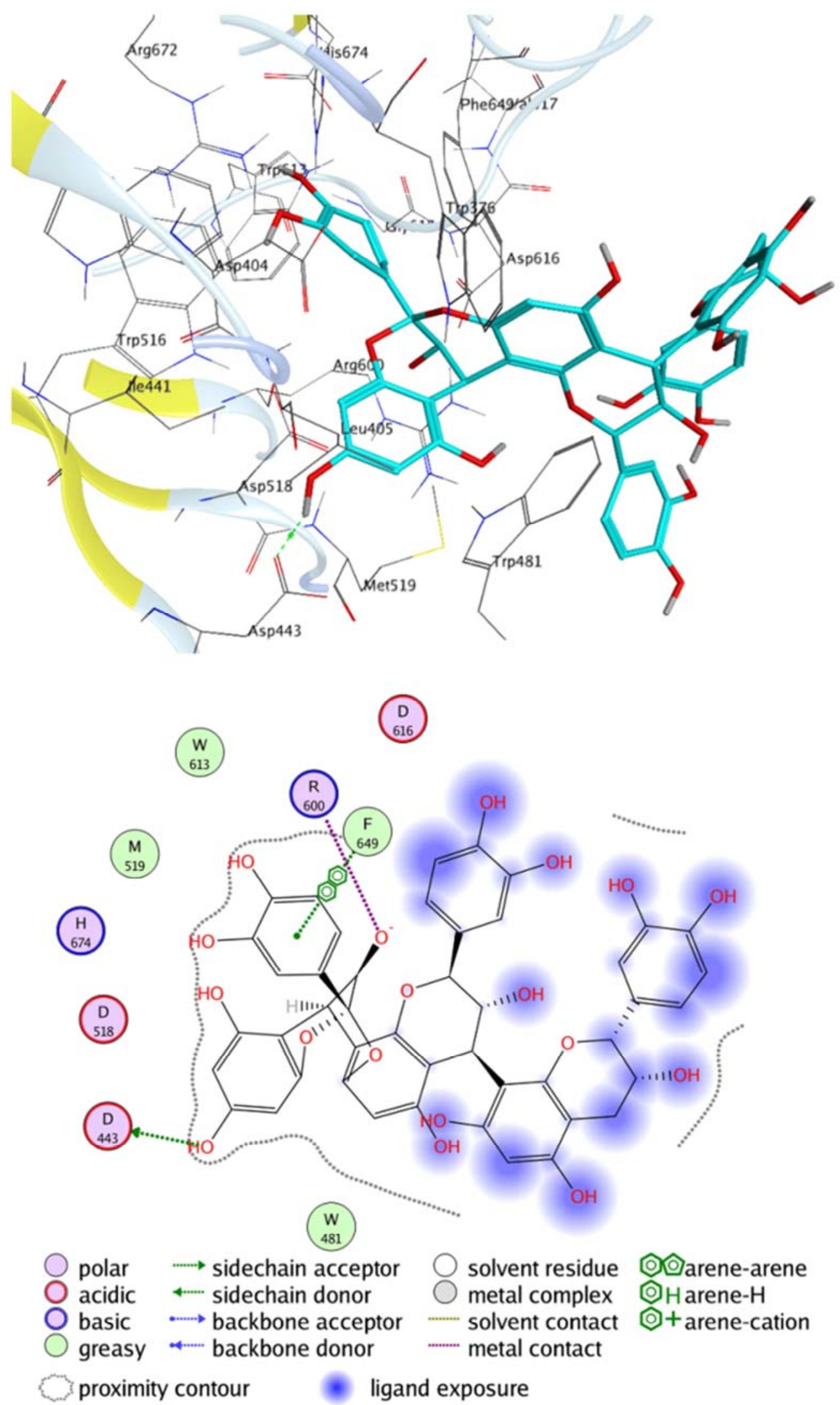

Figure A1. 3D- (with ribbon) and 2D-depiction of molecular docking of aesculitannin B (2) to the constructed $\alpha$-glucosidase. 
(A)

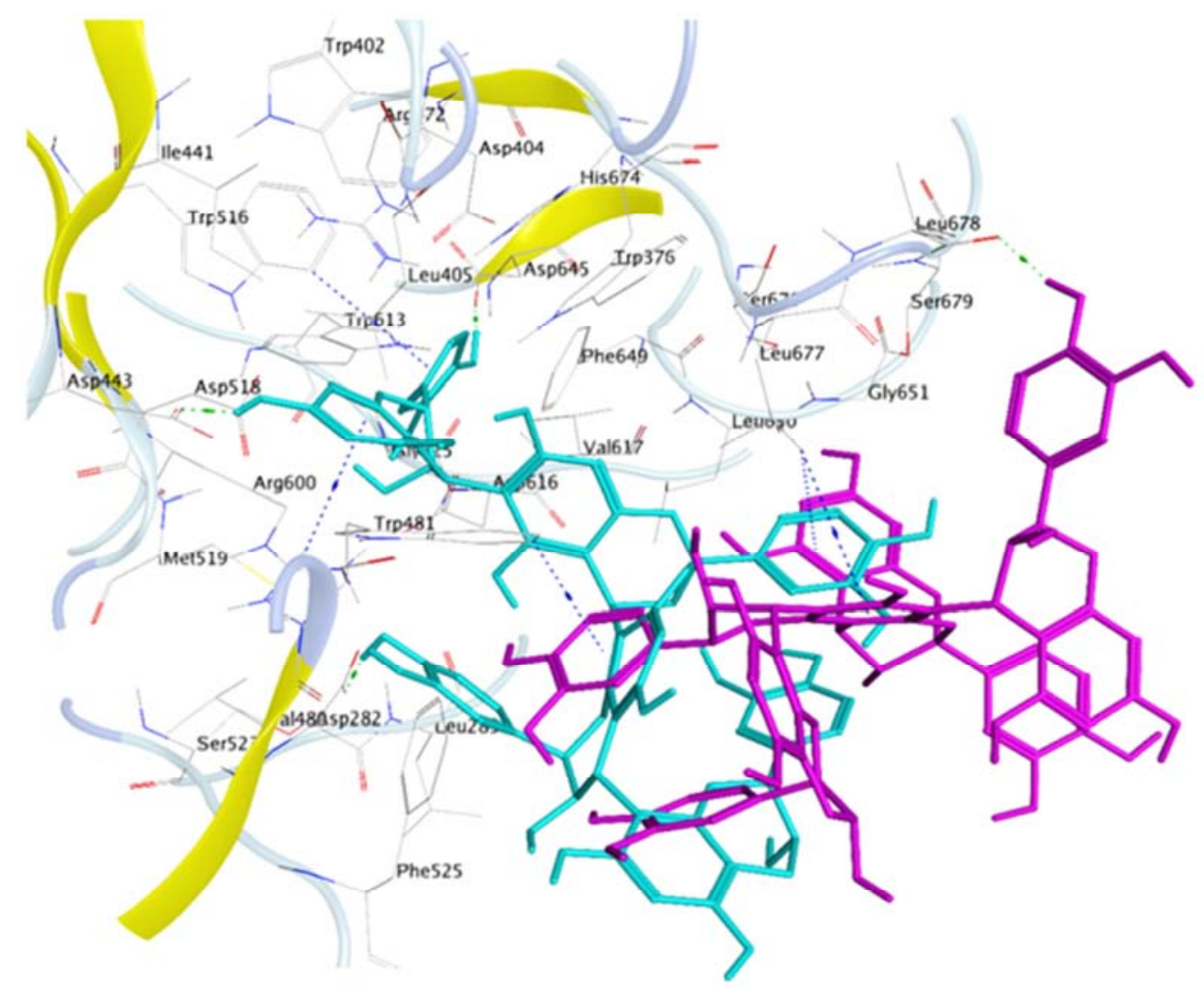

(B)

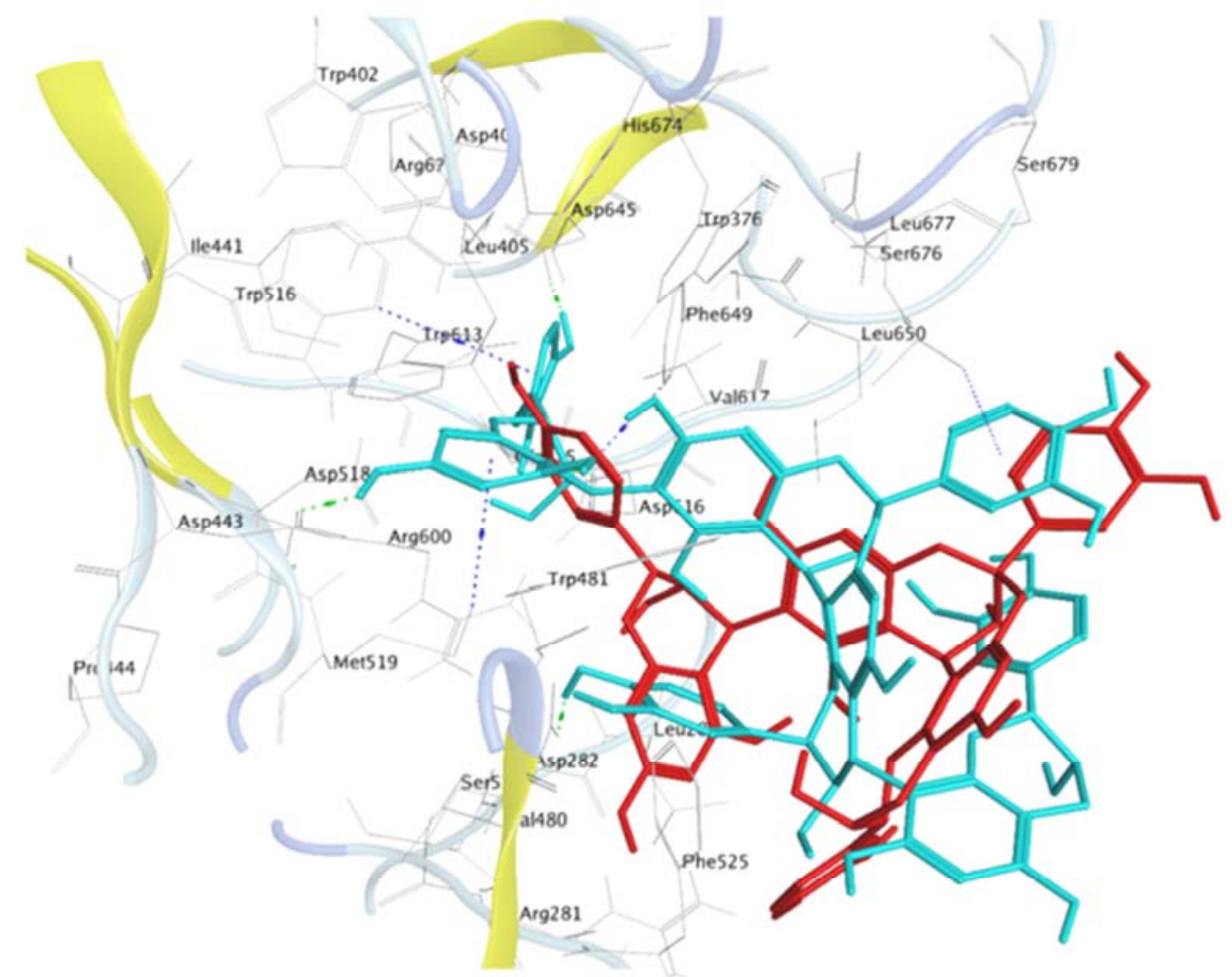

Figure A2. 3D-depiction (with ribbon) of molecular docking of oligo proanthocyanidins 5 (pavetannin C-1) versus 6 (parameritannin A-1) (A: 5, light blue; 6 , purple), and 5 versus 5-nor (B: 5, light blue; 5-nor, red) to the constructed $\alpha$-glucosidase model. 

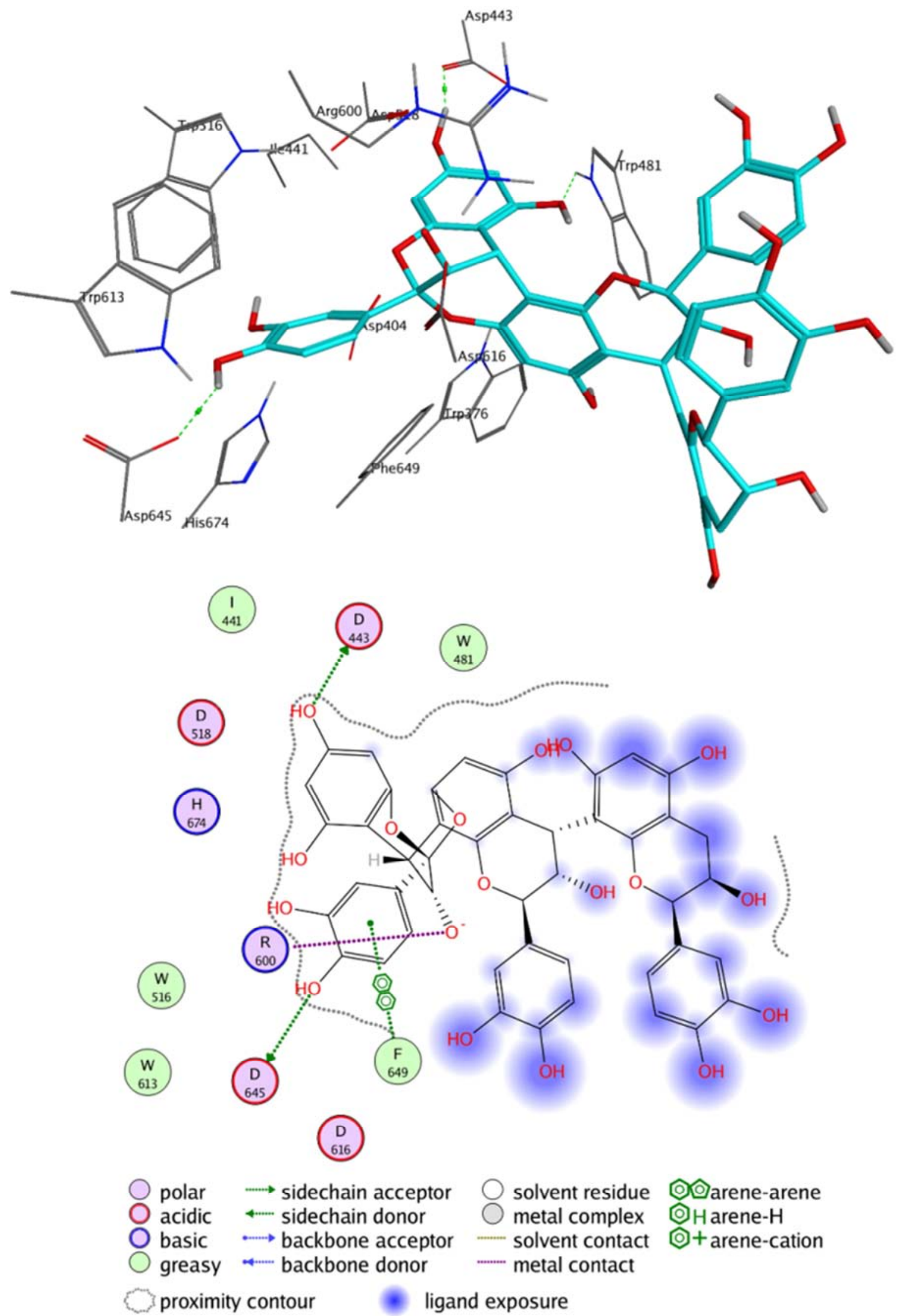

Figure A3. 2D- and 3D-depiction of molecular docking of trimeric proanthocyanidin 3-entC to the constructed $\alpha$-glucosidase. 


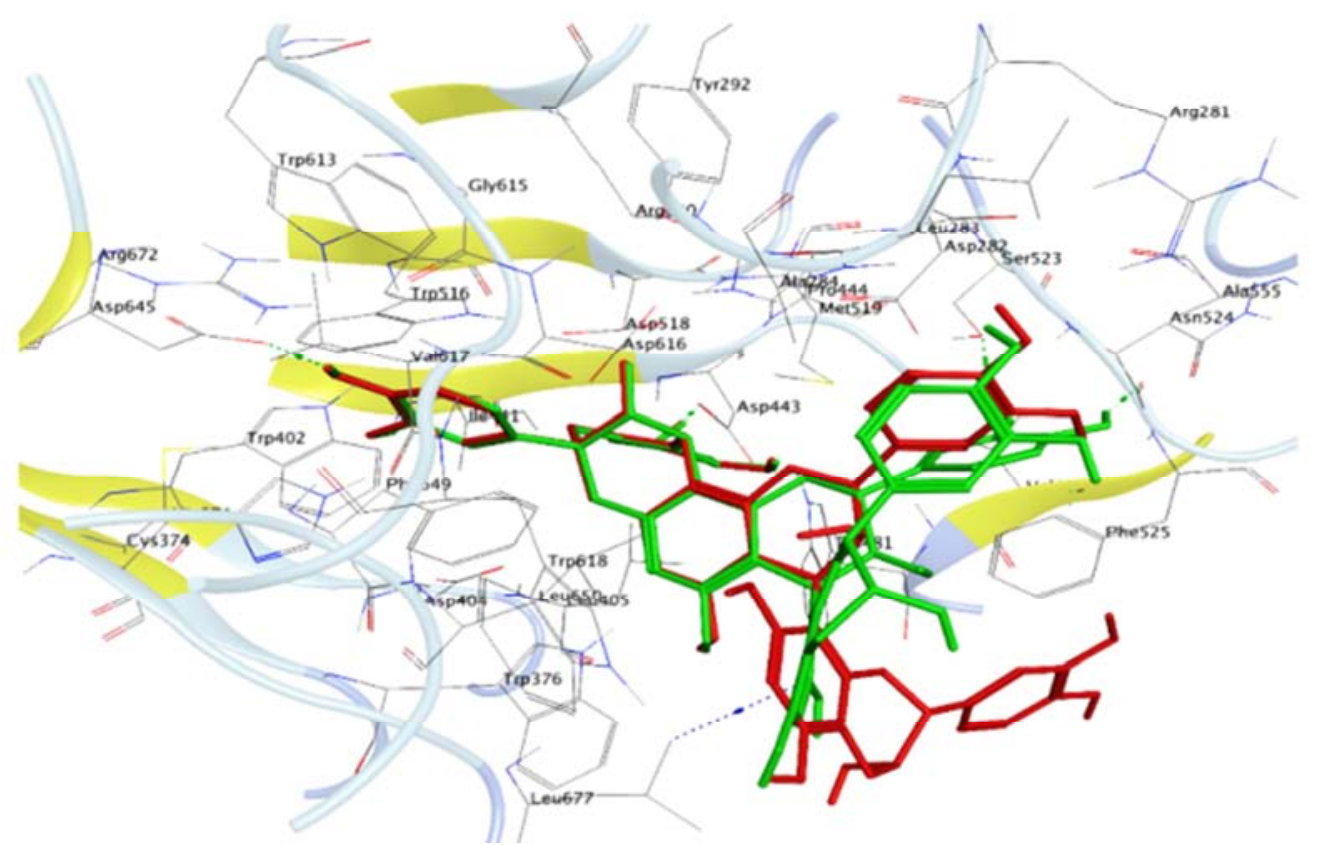

Figure A4. 3D-depiction (with ribbon) of molecular docking of trimeric proanthocyanidins $3 C$ (green) versus 3 ent (red) to the constructed $\alpha$-glucosidase model.
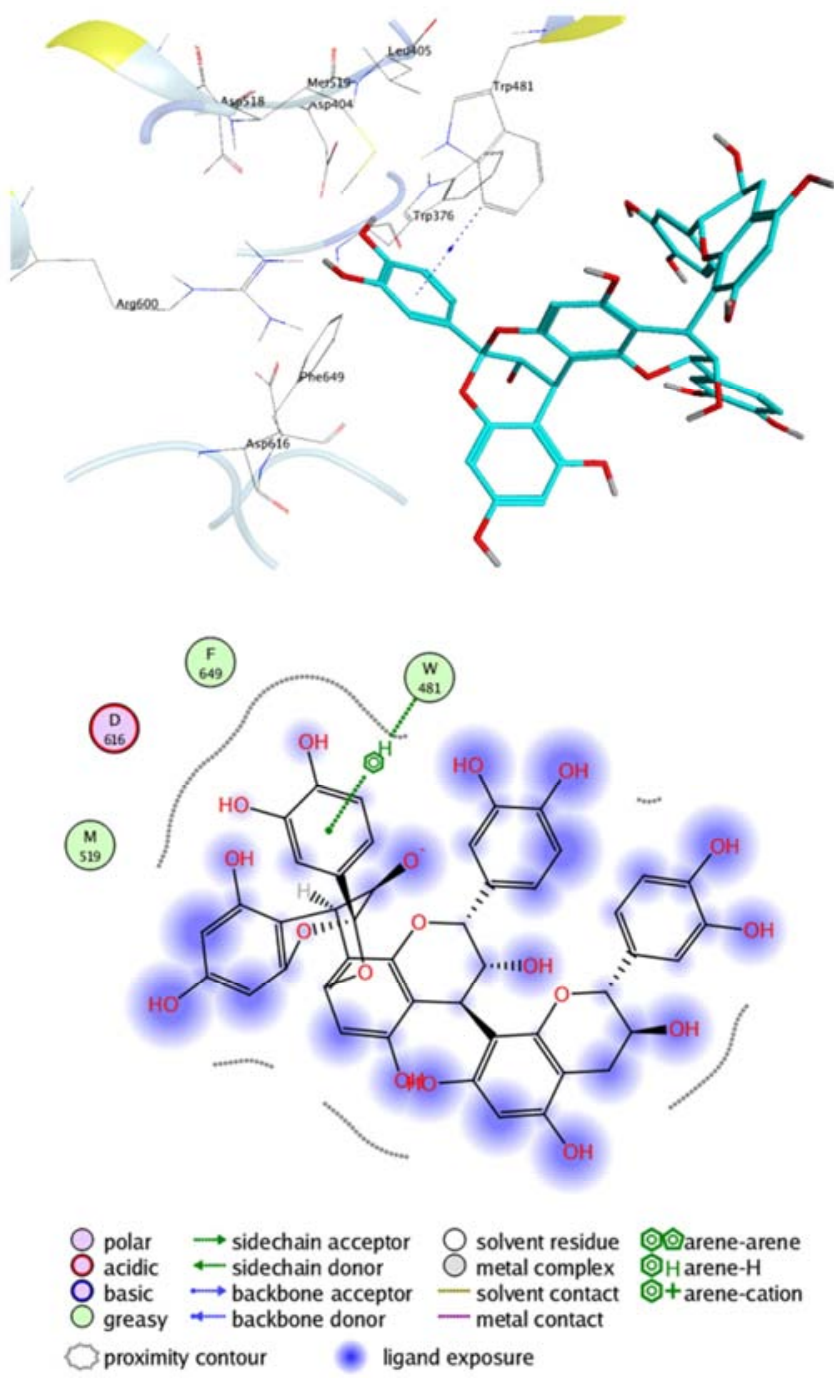

Figure A5. 3D- (with ribbon) and 2D-depiction of molecular docking of cinnamtannin D-1 (4) to the constructed $\alpha$-glucosidase. 


\section{References}

[1] S. Chiba (1997) Molecular mechanism in $\alpha$-glucosidase and glucoamylase. Bioscience, Biotechnology, and Biochemistry $61,1233-1239$.

[2] Nichols, B. L., J. Eldering, S. Avery, D. Hahn, A. Quaroni, and E. Sterchi (1998). Human small intestinal maltase-glucoamylase cDNA cloning homology to sucrase-isomaltase. Journal of Biological Chemistry 273, 3076-3081.

[3] G. Semenza and S. Auricchio, "Small intestinal disaccharidases," in The Metabolic Basis of Inherited Disease, $6^{\text {th }}$ ed. C. R. Scriver, A. L. Beaudet, A. S. Sly, D. Valle, J. B. Stanbury, J. B. Wyngaarden, and D. S. Fredrickson, Eds. New York: McGraw-Hill, 1989, pp 2975-2997.

[4] M. Hanefeld (1998) The role of acarbose in the treatment of non-insulin-dependent diabetes mellitus. Journal of Diabetes Complications 12, 228-237.

[5] Khan, M., M. Yousaf, A. Wadood, M. Junaid, M. Ashraf, U. Alam, et al. (2014) Discovery of novel oxindole derivatives as potent $\alpha$-glucosidase inhibitors. Bioorganic and Medicinal Chemistry 22, 3441-3448.

[6] Khan, K. M., F. Rahim, A. Wadood, N. Kosar, M. Taha, S. Lalani, et al. (2014) Synthesis and molecular docking studies of potent $\alpha$-glucosidase inhibitors based on biscoumarin skeleton. European Journal of Medicinal Chemistry 81, 245-252.

[7] Chang, C. C., S. L. Ho, and S. S. Lee (2015) Acylated glucosylflavones as $\alpha$-glucosidase inhibitors from Tinospora crispa leaf. Bioorganic and Medicinal Chemistry 23, 3388-3396.

[8] Lin, H. C. and S. S. Lee (2010) Proanthocyanidins from the Leaves of Machilus philippinensis. Journal of Natural Products 73, 1375-1380.

[9] Lou, H., Y. Ymazaki, T. Sasaki, M. Uchida, H. Tanaka, and S. Oka (1999) A-type proanthocyanidins from peanut skins. Phytochemistry 51, 297-308.

[10] Berman, H. M., J. Westbrook, Z. Feng, G. Gilliland, T. N. Bhat, H. Weissig, I. N. Shindyalov, and P. E. Bourne (2000) The Protein Data Bank. Nucleic Acids Research 28, 235-242.

[11] Vilar, S., G. Cozza, and S. Moro (2008) Medicinal chemistry and the molecular operating environment (MOE): application of QSAR and molecular docking to drug discovery. Current Topics of Medicinal Chemistry 8, 1555-1572.

[12] Davies, G. and B. Henrissat (1995) Structures and mechanisms of glycosyl hydrolases. Structure 3, 853-859.
[13] Lovering, A. L., S. S. Lee, Y. W. Kim, S. G. Withers, and N. C. J. Strynadka (2005) Mechanistic and structural analysis of a family 31 alpha-glycosidase and its glycosyl-enzyme intermediate. Journal of Biological Chemistry 280, 2105-2115.

[14] Sim, L., K. Jayakanthan, S. Mohan, R. Nasi, B. D. Johnston, B. M. Pinto, and D. R. Rose (2010) New glucosidase inhibitors from an Ayurvedic herbal treatment for type 2 diabetes: structures and inhibition of human intestinal maltase-glucoamylase with compounds from Salacia reticulata. Biochemistry 49, 443-451.

[15] P. Labute (2009) Protonate3D: assignment of ionization states and hydrogen coordinates to macromolecular structures. Proteins $75,187-205$.

[16] (a) T. A. Halgren (1996) Merck molecular force field. I. Basis, form, scope, parameterization, and performance of MMFF94. Journal of Computation Chemistry 17, 490-519; (b) ibid. (1999) MMFF VI. MMFF94s option for energy minimization studies. ibid. 20, 720-729.

[17] Santos-Buelgaa, C., Kolodzieij, H., and Treutter, D. (1995) Procyanidin trimers possessing a doubly linked structure from Aesculus hippocastanum. Phytochemistry 38, 499-504.

[18] Zhang, C. F., Sun, Q. S., Wang, Z. T., and Hattori, M. (2003) One new A-type proanthocyanidin trimer from Lindera aggregate (Sims) Kosterm. Chinese Chemical Letters 14, 1033-1036.

[19] Yoshimizu, M., Tajima, Y., Matsuzawa, F., Aikawa, S., Iwamoto, K., Kobayashi, T. et al. (2008) Binding parameters and thermodynamics of the interaction of iminosugars with a recombinant human acid alpha-glucosidase (alglucosidase alfa) insight into the complex formation mechanism. Clinica Chimica Acta 391, 68-73.

[20] Hermans, M. M. P., M. A. Kroos, J. van Beeurnen, B. A. Oostra, and A. J. Reuser (1991) Human lysosomal -glucosidase characterization of the catalytic site. Journal of Biological Chemistry 266, 13507-13512.

[21] Sugawara, K, K. Ohno, S. Saito, and Sakuraba H (2008) Structural characterization of mutant alpha-galactosidases causing Fabry disease. Journal of Human Genetics 53, 812-824.

[22] Sugawara, K., S. Saito, M. Sekijima, K. Ohno, Y. Tajima, and M. A. Kroos (2009) Structural modeling of mutant -glucosidases resulting in a processing/transport defect in Pompe disease. Journal of Human Genetics 54, 324-330.

[23] Perica, T. and C. Chothia (2010) Ubiquitin-molecular mechanisms for recognition of different structures. Current Opinion in Structural Biology 20, 367-376. 